\title{
The parental source of heteroploidy in chick embryos determined with chromosomally marked gametes
}

\author{
N. S. Fechheimer* and R. G. Jaap $\dagger$ \\ Departments of ${ }^{*}$ Dairy Science, $\dagger$ Poultry Science, and ${ }^{*} \dagger$ Genetics, The Ohio State University and \\ Ohio Agricultural Research and Development Center, Columbus, Ohio 43210, U.S.A.
}

\begin{abstract}
Summary. Males homozygous for chromosomal translocations were mated to karyologically normal females. The resulting embryos, after incubation for 16-18 h, were prepared for cytogenetic analysis. Of the 692 embryos analysed, $54(7.8 \%)$ were heteroploid or contained a major heteroploid cell line. Each of the 638 normal $2 n$ embryos contained one marker and one normal chromosome, indicating that nonearose from gynogenesis. Thirteen homogeneous haploid embryos were identified; 24 embryos were chimaeric $1 n / 2 n$ and one was $1 n / 3 n$. All haploid cell lines contained a marker chromosome indicating androgenetic origins. The 9 homogeneous triploid $(3 n)$ embryos and the $3 n$ cell line in a single $1 n / 3 n$ embryo contained a single marker. All resulted from fertilization by single spermatozoon of eggs that were diploid as a result of suppression of the second meiotic division. The $3 n$ lines of two $2 n / 3 n$ embryos were derived from other mechanisms. A single homogeneous tetraploid $(4 n)$ embryo and the $4 n$ cell lines of three $2 n / 4 n$ mosaic embryos each contained two marker chromosomes and presumably resulted from failure of cytokinesis in an early cleavage division.
\end{abstract}

\section{Introduction}

In a number of vertebrate species, including man, mouse, hamster, rat, rabbit, pig and chicken, the occurrence of heteroploid embryos is a primary contributing factor to embryonic death and wastage. Errors of meiosis, fertilization or early cleavage divisions may each be involved in the origin of one or more of the prevalent types of heteroploidy. A first step towards understanding of the situation is to determine at which stages in the reproductive process the errors are occurring that result in chromosomally abnormal embryos.

The domestic chicken has proved to be a particularly useful animal for the study of embryonic heteroploidy of types such as haploidy, triploidy, tetraploidy, euploid mosaicism and trisomy and monosomy (Bloom, 1972, 1974; Miller, Fechheimer \& Jaap, 1971, 1976; Snyder, Fechheimer \& Jaap, 1975). From the accumulated data it has been possible to infer the origins and causal factors of the most frequently occurring abnormalities (Bloom, 1974; Snyder et al., 1975). Tests of these inferences became possible with the availability of stocks bearing chromosomal translocations which can be made homozygous and used as 'markers' (Zartman \& Smith, 1975). This paper reports a study with embryos derived from parents of which one was homozygous for a marker chromosome.

\section{Materials and Methods}

Animals. The 28 females used were drawn from a stock of broiler-type chickens (AG), maintained at the Ohio State University, which had been shown previously to yield a high frequency of heteroploid embryos (Miller et al., 1971). The males used were homozygous for one of two chromosomal rearrangements: the two variant karyotypes and a normal one are shown in Pl. 1, Figs 1, 2 and 3. The complete description of these two translocations $(\mathrm{t}(\mathrm{OH} 4)$ and $\mathrm{t}(\mathrm{OH} 14))$ is given by Wooster, Fechheimer \& Jaap (1977). In this paper, the chromosomes with translocations are referred to as 'markers'. Only the male parents carried the marker chromosomes and each cock was homozygous for one marker. The genetic background of these males was primarily from a leghorn (egg-laying) type 
strain and the generation of males used in this experiment was derived from an outcross, two generations back, to the same broiler stock from which the females were derived. Their genome was therefore comprised of $75 \%$ Leghorn and $25 \%$ broiler.

Procedures. All birds were individually caged. Hens were artificially inseminated weekly with semen collected from the males by the massage method (Lake, 1956). Eggs were collected daily, identified, stored for up to 4 days at $10^{\circ} \mathrm{C}$ and incubated for $16-18 \mathrm{~h}$ at $37.8^{\circ} \mathrm{C}$. Preparations for karyological examination were made from the embryos by the procedure described by Miller et al. (1971). Each slide, containing cells from a single embryo, was scanned until 3 intact metaphase cells were found and these were analysed for the number of chromosomes of each of the nine major pairs. When one or more cells with a heteroploid complement was encountered, additional cells were analysed to a maximum of 30 . Any embryo for which an unambiguous analysis of a minimum of three cells could not be made was excluded from the results.

\section{Results}

Twenty-eight hens yielded 854 eggs ( $24.7 \pm 8.69$ (s.d.) eggs/hen). Karyological analysis of 692 embryos $(81 \%)$ showed that $54(7.8 \%)$ were heteroploid or contained a heteroploid cell line. Of the 162 eggs not analysed, 67 were infertile and 95 gave unusable preparations. The 638 normal diploid $(2 n)$ embryos all contained a single marker chromosome, indicating that each arose from a normal ovum fertilized by a normal spermatozoon. Not a single case of gynogenesis was found. The 54 aberrant embryos and details of the chromosomal abnormalities they contained are listed in Table 1.

Haploidy. Thirteen embryos were pure haploid $(1 n), 24$ were haploid-diploid $(1 n / 2 n)$ and one was a haploid-triploid $(1 n / 3 n)$ chimaera. The distribution of the 38 embryos with haploid cell lines among the 28 hens is shown in Table 2. Each of the 38 haploid cell lines contained a $\mathrm{Z}$ gonosome and a marker chromosome (Pl. 1, Fig. 4). All haploid cell lines were apparently derived from spermatozoa and not from ova. The $2 n$ cell lines of 23 of the $241 n / 2 n$ mosaic embryos contained a single marker chromosome; 12 contained the $\mathrm{ZW}$ sex chromosome complement, 9 were $\mathrm{ZZ}$ and in two the second gonosome could not be absolutely determined but was probably a $\mathrm{W}$. The proportions of $2 n$ cell lines with $\mathrm{ZZ}$ and $\mathrm{ZW}$ sex chromosome complements, and the presence of a single marker chromosome in $2 n$ cell lines of $1 n / 2 n$ embryos, indicates that all arose independently of the $1 n$ cell lines in the same embryos. Three gametes were apparently involved in the origin of these $231 n / 2 n$ embryos. One $1 n / 2 n$ embryo contained two markers and $\mathrm{ZZ}$ sex chromosomes in the $2 n$ line.

A single $1 n / 3 n$ mosaic embryo contained a single marker in each cell line; the haploid line therefore originated from a supernumerary spermatozoon while the triploid line resulted from three different gametic products in the same way as the pure triploids

Triploidy. In addition to the triploid cell line in the single $1 n / 3 n$ embryo, 9 pure triploids (Pl. 1 , Fig. 5) and 2 diploid-triploid ( $2 n / 3 n)$ mosaic embryos were found. Of the 9 pure $3 n$ embryos, 4 had sex

\section{EXPLANATION OF PLATE}

The figures represent partial karyotypes of individual chicken parents or embryos. Autosomal pairs 1-8 and the gonosomes are shown; the small autosomal pairs 9-38 are not shown.

Fig. 1. Partial karyotype of a normal female used as a parent.

Fig. 2. Partial karyotype of a male parent homozygous for a translocation $\mathrm{t}(\mathrm{OH} 4)$. Note the morphology of chromosome 2 compared with that in Fig. 1.

Fig. 3. Partial karyotype of a male parent homozygous for translocation $t(\mathrm{OH} 14)$. Note the altered morphology of chromosomes 2 and 4 compared to those in Fig. 1.

Fig. 4. Partial karyotype of a haploid embryo. Chromosomes 2 and 4 were derived from a male parent with a karyotype similar to that shown in Fig. 3.

Fig. 5. Partial karyotype of a triploid embryo. Of the three number 2 chromosomes, one was derived from a parent with karyotype similar to that shown in Fig. 2.

Fig. 6. Partial karyotype of a tetraploid cell from a $2 n / 4 n$ embryo. Two of the number 2 chromosomes are similar to those shown in Fig. 2 and two are as shown'lin Fig. 1. rom Bioscientifica.com at 04/26/2023 10:14:40AM 


\section{PLATE 1}

Fig.

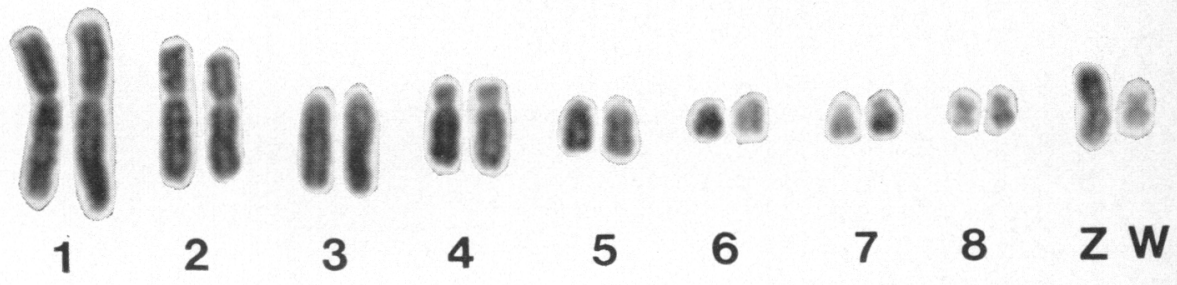

(4)

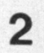

1 
Table 1. Numbers of heteroploid chicken embryos and their chromosome complements

\begin{tabular}{|c|c|c|c|c|}
\hline Type & Number & $\begin{array}{l}\text { Frequency } \\
\text { (no./1000) }\end{array}$ & $\begin{array}{l}\text { No. with various } \\
\text { complements* }\end{array}$ & $\begin{array}{l}\text { No. of 'marker' } \\
\text { chromosomes in } \\
\text { each cell line }\end{array}$ \\
\hline \multicolumn{5}{|l|}{ Haploid } \\
\hline $1 n$ & 13 & 19 & $\begin{array}{l}121 \mathrm{~A} \cdot \mathrm{Z} \\
11 \mathrm{~A} \cdot \mathrm{Z}+1\end{array}$ & $\begin{array}{l}1 \\
1\end{array}$ \\
\hline $1 n / 2 n$ & 24 & 35 & $\begin{array}{c}12 \text { 1A.Z/2A.ZW } \\
91 \mathrm{~A} . \mathrm{Z} / 2 \mathrm{~A} . \mathrm{ZZ} \\
2 \text { 1A.Z/2A.Z? } \\
1 \text { 1A.Z/2A.ZZ }\end{array}$ & $\begin{array}{l}1 / 1 \\
1 / 1 \\
1 / 1 \\
1 / 2\end{array}$ \\
\hline $1 n / 3 n$ & 1 & $1 \cdot 5$ & $11 \mathrm{~A} . \mathrm{Z} / 1 \mathrm{~A} . \mathrm{ZZZ}$ & $1 / 1$ \\
\hline \multicolumn{5}{|l|}{ Polyploid } \\
\hline $3 n$ & 9 & 13 & $\begin{array}{l}43 \mathrm{~A} . \mathrm{ZZZ} \\
53 \mathrm{~A} . \mathrm{ZWW}\end{array}$ & $\begin{array}{l}1 \\
1\end{array}$ \\
\hline $2 n / 3 n$ & 2 & $2 \cdot 9$ & $\begin{array}{l}12 \mathrm{~A} . Z W / 3 \mathrm{~A} . Z Z W \\
12 \mathrm{~A} . Z Z / 3 \mathrm{~A} . Z Z Z\end{array}$ & $\begin{array}{l}1 / 1 \\
1 / 2\end{array}$ \\
\hline $4 n$ & 1 & $1 \cdot 5$ & 1 4A.ZZWW & 2 \\
\hline $2 n / 4 n$ & 3 & $4 \cdot 3$ & $\begin{array}{l}2 \mathrm{2A} . Z Z / 4 A . Z Z Z Z \\
12 \mathrm{~A} . Z W / 4 \mathrm{~A} . Z Z W W\end{array}$ & $\begin{array}{l}1 / 2 \\
1 / 2\end{array}$ \\
\hline $\begin{array}{l}\text { Aneuploid } \\
2 n-1\end{array}$ & 1 & $1 \cdot 5$ & $12 \mathrm{~A} \cdot \mathrm{ZZ}-1,+$ frag. & 1 \\
\hline
\end{tabular}

${ }^{*}$ In this column $\mathrm{A}$ represents sets of autosomes, $\mathrm{Z}$ and $\mathrm{W}$ represent individual sex chromosomes. Slash $(/)$ separates two cell lines of the same embryo.

Table 2. Distribution of chick embryos with haploid or triploid cell lines among hens

\begin{tabular}{ccc}
\hline No. of hens & No. of embryos & $\begin{array}{c}\text { Mean no. of } \\
\text { embryos/hen }\end{array}$ \\
\hline Haploid & & \\
15 & 0 & $22 \cdot 9$ \\
2 & 1 & $24 \cdot 0$ \\
9 & 2 & $28 \cdot 1$ \\
1 & 4 & $18 \cdot 0$ \\
1 & 11 & $33 \cdot 0$ \\
\hline Triploid & & \\
20 & 0 & $24 \cdot 7$ \\
6 & 1 & $26 \cdot 7$ \\
2 & 3 & $19 \cdot 0$ \\
\hline
\end{tabular}

chromosomes ZZZ and 5 had ZWW. Each contained only a single marker chromosome and thus only a single set of paternal chromosomes, two sets having been derived from the hen.

One of the $2 n / 3 n$ embryos had sex chromosome ZW/ZZW and a single marker in each cell line. The other had sex chromosomes ZZ/ZZZ, a single marker in the $2 n$ line but two markers in the $3 n$ line, indicating two paternal chromosome sets in the $3 n$ line.

Tetraploidy. A single pure tetraploid ( $4 n$ : Pl. 1, Fig. 6) embryo and three $2 n / 4 n$ mosaics were observed. The four $4 n$ cell lines contained two markers and the three $2 n$ lines one marker. The sex chromosome complements of the $4 n$ lines of the mosaic embryos were $\mathrm{ZZ} / \mathrm{ZZZZ}$ and ZW/ZZWW, i.e. double that in the $2 n$ lines. Tetraploidy appears to have resulted from failure of cytokinesis of normal $2 n$ blastomeres. Failure of cytokinesis at first cleavage may have been the origin of the pure tetraploid. 
Aneuploidy. One aneuploid embryo was scored and is listed at the end of Table 1. Neither marker chromosome nor sex chromosome analysis are instructive of the origin of the error.

\section{Discussion}

The domestic chicken has proved to be particularly useful for elucidating some of the causal elements of chromosomal abnormalities. In man and other mammals the occurrence of heteroploid chromosome complements in embryos is perhaps the most important cause of abortion or embryonic death: it has been estimated that $60 \%$ of spontaneous abortions are the direct result of chromosomal abnormalities of the afflicted embryos (see Boué, Boué \& Lazar, 1975, for basis of this estimate). Chromosomal analysis of preimplantation mouse, rabbit, hamster and rat embryos also shows that a relatively high proportion die early because of chromosomal abnormality (Butcher \& Fugo, 1967; Vickers, 1969; Shaver \& Carr, 1969; Kaufman, 1973; Yamamoto, 1973; Fechheimer \& Beatty, 1974). Each species exhibits a unique array of heteroploid types reflecting its individual susceptibilities to errors of the reproductive process which result in chromosomal abnormalities (Ford, 1975). For example, in man there is a high frequency of aneuploidy, while the common laboratory mammals appear to be more inclined to errors of fertilization and of early cleavage division mitosis which result in a high frequency of polyploidy and mixoploidy.

Chicken embryos from different genetic lines show a different incidence of various forms of heteroploidy (Fechheimer, Miller \& Boerger, 1972; Bloom, 1972, 1974; Snyder et al., 1975). They display all the forms commonly seen in mammals, but haploidy, which is rarely encountered in mammals, is the most predominant. The hens used in the present study represent a line of birds in which the incidence of heteroploidy, particularly haploidy, had been shown to be high (Miller et al., 1976).

Haploidy. Arguments supporting an hypothesis that the haploid cell lines in $1 n / 2 n, 1 n / 3 n$ and other mixoploid embryos are, for the most part, androgenetic have been given by Miller et al. (1971, 1976) and Snyder et al. (1975). Zartman \& Smith (1975) have also reported a $1 n / 3 n$ embryo in which the haploid line contained a marker chromosome derived from the sire. In the present experiment an additional 38 embryos with haploid cell lines, all of paternal origin, are described. Thirteen of these embryos were pure haploid. It is not known what happens to the female pronucleus in these cases: it may be fertilized normally and the resulting zygotic nucleus fails to undergo first cleavage division, or it may undergo cleavage divisions but at a slower rate than the proliferating supernumerary sperm pronucleus. When the embryo is prepared for karyological analysis at the primitive streak stage, the diploid cells, being relatively few in number, are not encountered.

Haploid cell lines of paternal origin were also seen in all of the $241 n / 2 n$ mixoploid embryos. In 23 of these the female pronucleus was apparently normally fertilized and the resulting $2 n$ nucleus underwent cleavage. One or more accessory spermatozoa, also present, did not engage in syngamy but gave rise to separate haploid cell lines and $1 n / 2 n$ chimaeric embryos. Failure of cytokinesis at one mitosis, or fusion of two haploid cells, might have been the mechanism for formation of the diploid androgenetic cell line in the $1 n / 2 n$ mosaic embryo derived entirely from paternal products. Such a mechanism was proposed by Bloom (1970).

The haploid cell line of the $1 n / 3 n$ embryo was also of paternal origin. The $1 n / 3 n$ chick embryo reported by Zartman \& Smith (1975) was similar to that found in this study in terms of the parental source of the various gametic products involved.

The overall frequency of haploidy amongst the hens in this study was $5.5 \%$. It is clear from inspection of the data in Table 2 that production of haploid embryos is dependent on either the genotype or some permanent environmentally induced characteristic of the hens, the effect of which is to initiate and sustain proliferation, by mitotic-like activity, of spermatozoa.

Triploidy. If centromeres of sister chromatids divide at second meiotic division (MII), suppression of MII yields diploid ova with ZZ or WW sex chromosomes. Fertilization then yields triploid (3n) zygotes with ZZZ and ZWW in equal members. Mong, Snyder, Fechheimer \& Jaap (1974) concluded that about $80 \%$ of $3 n$ chick embryos (54) arose by this routes The data of Bloom(11974) on 58 
triploid embryos were in close agreement. Most of the $3 n . Z Z W$ embryos are thought to result from failure of MI of oogenesis and very few from dispermy or diploid spermatozoa. The two triploid embryos (ZZW) described by Zartman \& Smith (1975) contained only a single paternally derived marker chromosome and therefore resulted from MI suppression in the female.

In the present series of 10 triploids (including the $3 n$ line of the $1 n / 3 n$ embryo), all contained a single marker chromosome and therefore, by inference, a single set of paternally contributed chromosomes. Equal numbers were ZWW and ZZZ and all 10 can be considered as resulting from suppression of MII.

The rarity of a diandric origin of triploidy in birds is in contrast to the situation in mammals. Beatty (1974) estimated, from the data of Carr (1971) on triploidy in human aborted fetuses, that $60 \%$ of triploidy in man was digynic and $40 \%$ diandric. The primary diandric mechanism was suggested as fertilization by diploid spermatozoa, dispermy being rare.

Clustering of triploid embryos from particular hens is demonstrated by the data in Table 2: 6 of the 10 triploid embryos (disregarding the two $2 n / 3 n$ embryos) were from only 2 hens. Bloom (1972) noted the same phenomenon and speculated that a genetic influence might be implicated.

Diploid-triploid chimaerism. Snyder et al. (1975) have discussed the possible origins of the 7 examples of this abnormality which were then known and a single mechanism of derivation from binucleated oocytes was postulated to account for all. Both nuclei undergo normal MI, but MII is suppressed in one nucleus resulting in an oocyte with a $1 n$ and a $2 n$ nucleus. Each is fertilized by a single haploid spermatozoon thus yielding $2 n / 3 n$ embryos. The sex chromosome complements of the $2 n$ lines may be $\mathrm{ZZ}$ or ZW and of the $3 n$ lines $\mathrm{ZZZ}$ or $\mathrm{ZWW}$. All of the reported embryos contained some combination of these permitted sex chromosome complements.

Neither of the $2 n / 3 n$ embryos encountered in the present study could have arisen from the proposed mechanism. One contained sex chromosomes $\mathrm{ZW} / \mathrm{ZZW}$; each cell line had a single marker indicating only one set of paternal chromosomes and the maternally contributed sex chromosomes were therefore $\mathrm{W} / \mathrm{ZW}$. The simplest explanation is failure of cytokinesis at MI yielding two nuclei in the egg, each with ZW, one of which does not undergo MII. The $1 n$ nucleus and the $2 n$ nucleus of the resulting egg are each fertilized by a single spermatozoon. Alternatively two nuclei may have been present in the egg before MI; if one of these did not form a first polar body (failure of MI) the result would be compatible with the cytological observations.

The second embryo was of the type ZZ/ZZZ but the $3 n$ line contained two sets of paternal chromosomes. The mechanism responsible therefore yielded two separate $1 n$ nuclei in the egg, one of which was fertilized by a single spermatozoon and the other by two $1 n$ or one $2 n$ spermatozoa. Series of events such as these have been proposed to account for the occurrence of $2 n / 3 n$ mammalian embryos (Austin, 1961; Fechheimer \& Beatty, 1974). Clearly no single mechanism is sufficient to account for the various types of $2 n / 3 n$ chick embryos which have so far been reported. The 9 for which sex chromosome complements have been reported (including the 2 of this experiment) must have resulted from at least two different types of cytological error.

Tetraploidy. The observations on the embryos with $4 n$ cell lines in this study are compatible with and tend to verify the hypothesis (Miller et al., 1971, 1976; Snyder et al., 1975) that $4 n$ cell lines result from failure of cytokinesis at a cleavage division of a normal $2 n$ egg. The same phenomenon (at different ploidy levels) apparently occurred to produce one of the $1 n / 2 n$ mosaic embryos of the present report and a $3 n / 6 n$ mosaic reported by Miller et al. (1971). When the failure occurs at the first cleavage division (of a normal $2 n$ egg) the result is a pure $4 n$ embryo. Other possible mechanisms that may give rise to $4 n$ embryos are the occurrence and parthenogenetic development of completely unreduced eggs or the fertilization of a $1 n$ egg by three spermatozoa. No evidence is available in support of the latter mechanism. The finding of two pentaploid ( $5 n$ ) embryos (Miller et al., 1976; N.S. Fechheimer, unpublished) with sex chromosome complements ZZZWW support the contention that $4 n$ eggs do occasionally occur and parthenogenesis has been shown to occur in at least some lines of chickens (Olsen, 1967; Olsen, Wilson \& Marks, 1968).

Miss S. L. Woolard provided excellent technical assistance. The paper is approved as Journal Article No. 94-77 of the Ohio Agricultural Research and Development Center. ica.com at 04/26/2023 10:14:40AM 


\section{References}

Austin, C.R. (1961) The Mammalian Egg. Blackwell Scientific Publications, Oxford.

BEATTY, R.A. (1974) Genetic aspects of spermatozoa. In Physiology and Genetics of Reproduction, Part A, pp. 183-196, Eds E.M. Coutinho \& F. Fuchs. Plenum Press, New York.

BLoom, S.E. (1970) Haploid chicken embryos: evidence of diploid and triploid cell populations. J. Hered.61, 147-150.

BLoom, S.E. (1972) Chromosome abnormalities in chicken (Gallus domesticus) embryos: types, frequencies and phenotypic effects. Chromosoma 37, 309-326.

BLoom, S.E. (1974) The origins and phenotypic effects of chromosome abnormalities in avian embryos. In Proc. 15th Wld Poultry Congr., New Orleans, pp. 316-320. U.S.A. Branch of Wld. Poultry Sci. Assn., Washington, D.C.

Boué, J., Bout, A. \& Lazar, P. (1975) The epidemiology of human spontaneous abortions with chromosomal anomalies. In Aging Gametes, pp. 330-348. Ed. R.J. Blandau. S. Karger, Basel.

Butcher, R.L. \& Fugo, N.W. (1967) Overripeness of the mammalian ova. II. Delayed ovulation and chromosome anomalies. Fert. Steril. 18, 297-302.

CarR, D.H. (1971) Chromosome studies in selected spontaneous abortions: polyploidy in man. $J$. med. Genet. 8, 164-174.

FeChHeimer, N.S. \& Beatty, R.A. (1974) Chromosome abnormalities and sex ratio in rabbit blastocysts. $J$. Reprod. Fert. 37, 331-341.

FeChHeimer, N.S., MilleR, R.C. \& Boerger, K.P. (1972) Genetic influence on incidence and type of chromosome aberrations in early chicken embryos. Proc. 7th Int. Congr. Anim. Reprod. \& A.I., Munich, Vol. II, pp. 1129-1133.

FORD, C.E. (1975) The time in development at which gross genome unbalance is expressed. In The Early Development of Mammals, pp. 285-304. Eds M. Balls \& A. E. Wild. Cambridge University Press.

KaUfmaN, M.H. (1973) Analysis of the first cleavage division to determine the sex-ratio and incidence of chromosome anomalies at conception in the mouse. J. Reprod. Fert. 35, 67-72.
LAKE, P.E. (1956) Collection of fowl semen by the massage method. J. agric. Sci., Camb. 49, 120-126.

Miller, R.C., FeChHeimer, N.S. \& JAAP, R.G. (1971) Chromosome abnormalities in 16-18 hour chick embryos. Cytogenetics 10, 121-136.

Miller, R.C., FechHeimer, N.S. \& JAAP, R.G. (1976) Distribution of karyotype abnormalities in chick embryos sibships. Biol. Reprod. 16, 549-560.

MONG, S.J., SNYdER, M.D., FEChHEIMER, N.S. \& JAAP, R.G. (1974) The origin of triploidy in chick (Gallus domesticus) embryos. Canad. J. Genet. Cytol. 16, 317-322.

OLSEN, M.W. (1967) Early development of unfertilized turkey and chicken eggs. Wld's Poult. Sci. J. 23, 242245.

OzSEN, M.W., Wilson, S.P., \& MARKS, H.L. (1968) Genetic control of parthenogenesis in chickens. $J$. Hered. 59, 41-42.

Shaver, E.L. \& CARR, D.H. (1969) The chromosome complement of rabbit blastocysts in relation to the time of mating and ovulation. Can.J. Genet. Cytol. 11, 287-293.

SNyder, M.D., Fechheimer, N.S. \& JAAP, R.G. (1975) Incidence and origin of heteroploidy, especially haploidy in chick embryos from intraline and interline matings. Cytogenet. Cell Genet. 14, 6375.

VICKERS, A.D. (1969) Delayed fertilization and chromosomal anomalies in mouse embryos. J. Reprod. Fert. 20, 69-76.

Wooster, W.E., FechHeImer, N.S. \& JAAP, R.G. (1977) Structural rearrangements of chromosomes in the domestic chicken: experimental production by $\mathrm{X}$ irradiation of spermatozoa. Can. J. Genet. Cytol. 19, 437-446.

Y AмAMото, M. (1973) Maternal age dependence of chromosome anomalies. Nature, New Biol. 241, 141.

ZaRTMAN, D.L. \& SMith, A.L. (1975) Triploid and haploid-triploid mosaicism among chick embryos (Gallus domesticus). Cytogenet. Cell Genet. 15, 138145.

Received 16 June 1977 